\title{
Comparison of Several Hyperspectral Image Fusion Methods for Visualization
}

\author{
Hongwen Lin, Anqing Zhang, Shaoqing Yang \\ Department of Information Combat, Dalian Naval Academy, 116018, China
}

Keywords:hyperspectral image, visualisation, colour representation, comparison .

\begin{abstract}
Hyperspectral image visualization is an important research aspect in hyperspectral image fusion. This paper compared four typically used hyperspectral image visualization methods: method based on bilateral filter, method based on Principal Component Analysis (PCA), method based on independent component analysis (ICA) and method based on optimization. Fusion framework and scheme are explained briefly. Two sets of images obtained by AVIRIS and ROSIS sensors are used in our experiments, and four statistical assessment parameters, namely variance, entropy, average gradient and fusion factor are adopted to comparatively analyze the fusion results. The comparison results show that the effects of bilateral filter method, PCA method and optimization method are similar, and they are superior to ICA method.
\end{abstract}

\section{Introduction}

The research in the area of hyperspectral image processing is of growing interest due to its high spectral resolution, which contains around 200-250 images of the same scene. The hyperspectral image applications have been explored in various areas like, remote sensing, environment monitoring, geological surveying and surveillance. In hyperspectral data processing, data fusion is the hottest technique, which is a process merging two or more images in such a way as to retain the most desirable characteristics of each. Visualization of the hyperspectral scene is an important area in hyperspectral data fusion. However, it is not possible to directly display hyperspectral images with conventional displays that typically display color images using three bands, namely red, green and blue (RGB). Many strategies for hyperspectral image color display[1-6] have been proposed in recent years. In this paper, several of them are compared and quantitative analysis is taken to explain the effect of these methods.

The organization of this paper is as follows. In the next section, four typically used hyperspectral image visualization methods are depicted. Quantitative measures of fusion performance are described in section III. Two datasets are used to perform experiments, and qualitative and quantitative comparisons are done in section IV. At last conclusions are drawn in section V.

\section{Hyperspectral image visualization methods}

Typically used methods for hyperspectral image visualization includes method based on band selection[1,2], method based on weighting[3], method based on data projection[4,5] and method based on optimization[6]. However, methods based on band selection only select three bands out of hundreds of ones, and no data fusion operation is implemented, a high amount of information present in the input data is lost, so it is not considered in this paper. The other three categories of methods are described briefly as follows.

\subsection{Method based on Weighting}

This kind of techniques generate the resultant fused image as a linear combination of the input image bands to obtain an appropriate contribution from each of the input pixels. The weights may be fixed, and they may also be calculated from some measure that describes the relative importance of the given pixel or the band in the input data with reference to the local neighbourhood.

A methods based on bilateral filter is proposed in [3]. Let $I\left(x, y, \lambda_{1}\right){ }_{\text {to }} I\left(x, y, \lambda_{M}\right)$ be the subset of a hyperspectral image cube, containing $M$ images from consecutive wavelength bands $\lambda_{1}$ to $\lambda_{M}$. The 
method calculate the weight at each pixel $(x, y)$ for each image, ${ }^{w_{1}}$ to $w_{M}$, using the bilateral filter as shown in the following:

$$
\omega_{i}(x, y)=\frac{\left|I\left(x, y, \lambda_{i}\right)-I_{B F}\left(x, y, \lambda_{i}\right)\right|+K}{\sum_{i=1}^{M}\left(\left|I\left(x, y, \lambda_{i}\right)-I_{B F}\left(x, y, \lambda_{i}\right)\right|+K\right)}
$$

Where $I_{B F}$ is the corresponding bilateral filtered image. $\mathrm{K}$ is a positive real number that allows flexibility in the fusion process by increasing or decreasing the effect of actual weight components and prevents numerical instability at homogenous regions. The fused image of the hyperspectral cube subset $I_{F}$ is given by

$$
I_{F}(x, y)=\sum_{i=1}^{M} w_{i}(x, y) I\left(x, y, \lambda_{i}\right)
$$

\subsection{Method base on data projection}

Researchers have also looked at the problem of hyperspectral image visualization from the perspective of dimensionality reduction. Data projection is one of the common methods to reduce data dimensionality by projecting high-dimensional data to lower dimensions. principal component analysis (PCA) and independent component analysis (ICA) are representative methods.

i) Method based on PCA

Principal Component Analysis is a commonly used technique for colour display [4].

Let $\Sigma$ be sample covariance matrix for an L-band hyperspectral image, and let $V=\left[v_{1}, v_{2}, \cdots, v_{L}\right]$ and $\Lambda=\operatorname{diag}\left\{\lambda_{1}, \lambda_{2}, \cdots, \lambda_{L}\right\}$ be the eigenvector and eigenvalue matrices of $\Sigma$, which can be related as

Then the PC images can be calculated by

$$
V^{T} \Sigma V=\Lambda
$$

$$
Z_{P C A}=V^{T}(\mathbf{z}-\mathbf{m})
$$

where $\mathbf{m}$ is the sample mean vector, and $\mathbf{z}$ and $Z_{P C A}$ are pixels vectors before and after the transform, respectively.

The first principal component (PC) image is selected as the fused image.

ii) Method based on ICA

Independent component analysis is a statistical and computational technique that extracts independent source signals by searching for a linear or nonlinear transformation that minimizes the statistical dependence between components

The classical ICA model is given by $x=A s$, where $x$ is the vector of observed signals, $A$ is the mixing matrix, and $S$ is the vector of the original signals. The general idea behind ICA is to estimate the inverse matrix of $\mathrm{A}, W=A^{-1}$, and reconstruct $\mathrm{s}$ by $s=W x$. Three ICs are selected from all ICs for display.

A CCMI method for ICs selection using the correlation coefficient (CC) and Mutual information (MI) is presented in [5]. The correlation between the ith band image in $\mathrm{H}$ and the jth IC in A can be written as:

$$
C_{i j}=\frac{\sum_{m} \sum_{n}\left(H_{i}-\bar{H}_{i}\right)\left(A_{j}-\bar{A}_{j}\right)}{\sqrt{\left(\sum_{m} \sum_{n}\left(H_{i}-\bar{H}_{i}\right)^{2}\right)\left(\sum_{m} \sum_{n}\left(A_{j}-\bar{A}_{j}\right)^{2}\right)}}
$$

With $\bar{H}_{i}$ and $\bar{A}_{j}$ indicating the means of the ith and jth images in $H$ and $A$, respectively; and $C_{i j}$ is a real number between -1 and 1 .

The mutual information of the ith image in $\mathrm{H}$ and the jth component in $\mathrm{A}$ is defined as 


$$
I_{H_{i} A_{j}}=\sum_{n} \sum_{m} p_{H_{i} A_{j}}(m, n) \log \frac{p_{H_{i} A_{j}}(m, n)}{p_{H_{i}}(m) P_{A_{j}}(n)}
$$

$p_{H_{i}}(m)$ and $P_{A_{j}}(n)$ respectively represent the intensity values of the ith image in $\mathrm{H}$ and the jth component in A , and $p_{H_{i} A_{j}}(m, n)$ represents the joint probability of a pair of pixel intensities.

The CCMI method selects an IC which is most similar to the original hyperspectral image dataset and includes most of its information.

\subsection{Method based on optimization}

The idea of optimization-based technique is that some objective functions are established based on specific optimization criteria.

A method with entropy maximization, variance maximization and smoothness of weight as criterions is mentioned in [6]. The objective function is as follows:

$$
\begin{aligned}
& J(\alpha)=-\varepsilon_{1}(\alpha)-\lambda_{c} \varepsilon_{2}(\alpha)+\lambda_{s} \varepsilon_{3}(\alpha) \\
& \text { subject to } \alpha_{k}(x, y) \geq 0, \quad \forall k, \\
& \text { and } \sum_{k=1}^{B} \alpha_{k}(x, y)=1, \forall(x, y)
\end{aligned}
$$

Where $\varepsilon_{1}(\alpha)=-\int_{x} \int_{y} f(x, y) \log \left(\frac{f(x, y)}{0.5 e}\right) d x d y$ fulfils the entropy maximization criterion;

$\varepsilon_{2}(\alpha)=\frac{1}{X Y} \int_{x} \int_{y}\left(f(x, y)-\frac{\int_{x} \int_{y} f(x, y) d x d y}{X Y}\right)^{2} d x d y$ fulfils the variance maximization criterion;

$\varepsilon_{3}(\alpha)=\int_{x} \int_{y} \sum_{k=1}^{B}\left(\alpha_{k_{x}}^{2}(x, y)+\alpha_{k_{y}}^{2}(x, y)\right) d x d y$

The fused image is

fulfils the smoothness of the weight criterion.

$$
f(x, y)=\sum_{k=1}^{B} \alpha_{k}(x, y) i_{k}(x, y)
$$

where $i_{k}(x, y)$ represents the pixel at location $(x, y)$ in the kth band of the hyperspectral image, and $\alpha_{k}(x, y)$ acts as the weight for fusion. $\alpha_{k_{x}}(x, y)$ and $\alpha_{k_{y}}(x, y)$ denote differentiation in the respective directions.

The weights should satisfy the following properties:

$$
\sum_{k=1}^{B} \alpha_{k}(x, y)=1 \forall(x, y) \text { and } \alpha_{k}(x, y)>0 \forall(x, y) .
$$

\section{Quantitative measures of fusion performance}

Qualitative and quantitative inspections are two major means to evaluate the performance of distinct fusion schemes. However, qualitative approaches may contain subjective factors and can be influenced by personal preferences or eyesight. Due to these problems, quantitative approaches are often required and more desired to evaluate the experimental results. In this paper, we perform the performance evaluation on the basis of statistical assessment parameters presented in [7] and [8], which includes variance, entropy, average gradient and fusion factor.

\subsection{Variance}

The variance of an image, $\sigma^{2}=\operatorname{var}(I)$, is directly related to the image contrast. Variance measures the deviation of gray values of the pixels from the image mean. Images with higher variances have a better contrast, which makes visualization simple and appealing. However, the 
variance tends to be higher with addition of noise; therefore, a high value of variance need not necessarily imply a better quality.

\subsection{Entropy}

The entropy of an image describes the total amount of information contained in the image, which is calculated as

$$
H_{x}=-\sum_{x} p_{x}(x) \ln p_{x}(x)
$$

where $p_{x}(x)$ is the probability density of the intensity level $x$ in the image. An image consisting of a small number of intensity values has a low entropy value and presents very little information. On the other hand, an image with dispersed distribution has a high entropy value and contains a lot of information. Similar to the variance measure, image entropy is also susceptible to the presence of noise.

\subsection{Average gradient}

The average gradient of an image $\bar{g}$ is the measure of image sharpness in terms of gradient values. It helps human observers to identify various features, and also improves the performance of various machine vision algorithms such as image segmentation and object recognition. If $I_{x}$ denotes the difference in the x-axis direction and ${ }^{y}$ denotes the difference in the $\mathrm{y}$-axis direction for the image size $\mathrm{N}$, the average gradient is given by

$$
\bar{g}=\frac{1}{N} \sum_{x} \sum_{y} \sqrt{I_{x}^{2}+I_{y}^{2}}
$$

\subsection{Fusion factor}

The measure used to quantify the similarity between the final image and the constituent input image bands in terms of mutual information is known as the fusion factor (FF), which was mentioned in [9], In [8], a modified definition of the FF has been proposed that weighs contribution of the individual bands towards the result of fusion based on the information content within the band. If $M I\left(I_{i}, I_{f}\right)$ denotes the amount of mutual information between one of the input bands $I_{i}$, and the fused image generated by fusing bands $I_{f}$, then the fusion factor FF is given as follow ,

$$
F F=\left(\sum_{i=1}^{B} M I\left(I_{i}, I_{f}\right) H\left(I_{i}\right)\right) / \sum_{i=1}^{B} H\left(I_{i}\right)
$$

where $^{H\left(I_{i}\right)}$ is the entropy of the band. High values of this measure suggest a higher amount of mutual information between the resultant image and its constituent hyperspectral bands, is desirable.

\section{Comparisons of the fusion performance}

To verify the effectiveness of the techniques discussed above, two hyperspectral data sets were used. The first data set is taken over northwest Indiana's Indian Pine Test Site in June 1992 by AVIRIS. This data set consists of $145 \times 145$ pixels with 220 bands. The number of bands is initially reduced to 200 bands by removing bands covering water absorption and noisy bands. The second one is collected by the ROSIS optical sensor over the urban area of the University of Pavia, Italy, the image size in pixels is $610 \times 340$. The number of data channels is 103 . For the ease of computation, only top left corner of size $200 \times 200$ is used in our experiments. 


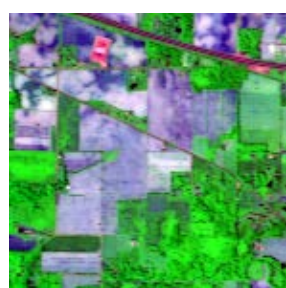

(a)

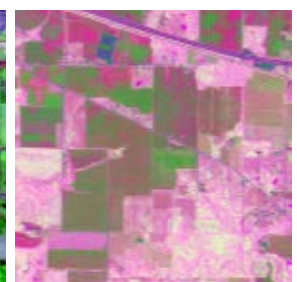

(b)

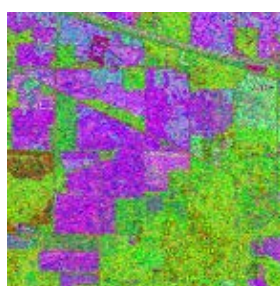

(c)

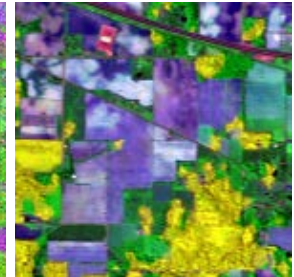

(d)

Fig.1: Experiment result of Indian Pines data set (a) Bilateral filter method; (b) PCA method; (c)

ICA method; (d) optimization method

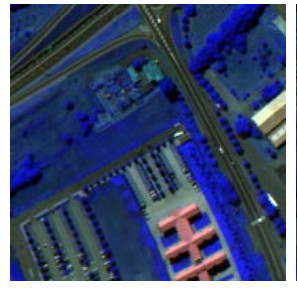

(a)

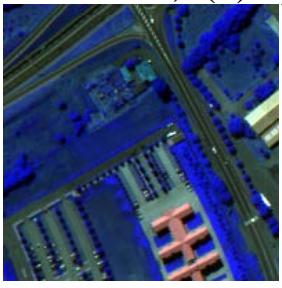

(b)

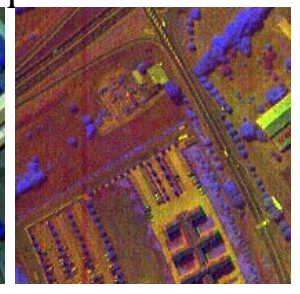

(c)

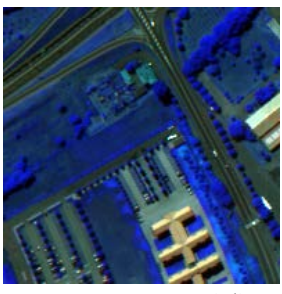

(d)

Fig.2: Experiment result of Pavia University data set (a) Bilateral filter method; (b) PCA method; (c) ICA method; (d) optimization method

To obtain a colour fused image, the original image is partitioned into three subgroups, fusion operation is implemented to each subgroup, and three fused image is obtained, then they are assigned as Red, Green and Blue components of the final fused colour image. There are many partitioning approaches, such as partitioning based on the RGB spectrum, equal subgroups partitioning, maximum energy partitioning, partitioning based on spectral signature, partitioning based on correlation coefficient, and etc. In this paper, we use correlation coefficient to partition the original image.

The experimental results are shown in Fig.1 and Fig.2 Fig.1 (a) and Fig.2(a) are the results of method based on bilateral filter respectively, Fig.1(b) and Fig.2(b) are the result of method based on PCA, Fig.1 (c) and Fig.2(c) are the result of method based on ICA, Fig.1(d) and Fig.2(d) represent the result of method based on optimization. It is easy to see that, the differences between bilateral filer method, PCA method and optimization method are slight if only judged by eyesight, especially to Pavia University scene data set, but they are superior to method based on ICA.

Quantitative comparisons are implemented and the results are shown in Table I and Table II for the Indian Pines data set and Pavia University data set respectively. The variance, entropy, average gradient and fusion factor are calculated using formulas described in section III for each colour components, and the means of each parameter are used for the final result. The best results for each quality measure are labeled in bold. Quantitative factors show that the performances of bilateral filter method, PCA method and optimization method are very close. Optimization method has the largest variance; PCA method has the largest fusion factor. ICA method has the largest average gradient, this is accord with the appearance of the result, because of the discontinuity of fused image. ICA method has the lowest entropy which means that the fusion results contain the least information.

Table 1: Quantitative assessment of various methods for Indian Pines data set

\begin{tabular}{ccccc}
\hline Method & Variance & Entropy & $\begin{array}{c}\text { Average } \\
\text { gradient }\end{array}$ & Fusion factor \\
\hline bilateral filtering method & 3804.54 & 7.65 & 131.58 & 1.80 \\
PCA method & 3787.51 & 7.68 & 123.22 & 2.08 \\
ICA method & 3858.06 & 7.44 & 166.92 & 1.00 \\
optimization method & 3914.03 & 7.73 & 140.32 & 1.64 \\
\hline
\end{tabular}


Table 2: Quantitative assessment of various methods for Pavia University data set

\begin{tabular}{ccccc}
\hline Method & Variance & Entropy & $\begin{array}{r}\text { Average } \\
\text { gradient }\end{array}$ & Fusion factor \\
\hline bilateral filtering method & 1593.93 & 6.99 & 90.36 & 2.10 \\
PCA method & 1585.50 & 6.95 & 89.38 & 2.19 \\
ICA method & 1261.68 & 6.53 & 111.4 & 1.13 \\
optimization method & 1627.09 & 6.93 & 89.97 & 1.99 \\
\hline
\end{tabular}

\section{Conclusions}

In this paper, we performed experiments to compare and analyze four types of hypersepctral image visualization methods. We explained the fusion framework and scheme of each technique briefly. Four statistical assessment parameters, namely variance, entropy, average gradient and fusion factor are adopted to compare the fusion results. Two hyperspectral data sets obtained by AVIRIS and ROSIS sensors are used for our experiments. Qualitative and quantitative comparisons show that the effects of method based on bilateral filer, method based on PCA and method based on optimization are very similar, but they are superior to method based on ICA.

\section{Acknowledgements}

This work is supported by Natural Science Foundation of China (No.601303192).

\section{References}

[1] Hongjun su, QianDu, Peijun Du. Hyperspectral Image Visualization Using Band Selection. IEEE Journal of Selected Topics in Applied Earth Observations and Remote Sensing. PP,1-12,2013

[2] Demir, B., Çelebi, A., Ertürk, S. A low-complexity approach for the color display of hyperspectral remote-sensing images using one-bit-transform-based band selection. IEEE Trans. Geosci. Remote Sens. 47(1), pp. 97-105,2009

[3] Ketan Kotwal, Subhasis Chaudhuri. Visualization of Hyperspectral Images Using Bilateral Filtering. IEEE Trans. Geosci. Remote Sensing, vol. 48,no. 5, pp. 2308-2316, May 2010

[4] V. Tsagaris, V. Anastassopoulos, and G. Lampropoulos. Fusion of hyperspectral data using segmented PCT for color representation and classification. IEEE Trans. Geosci. Remote Sens., vol. 43, no. 10,pp. 2365-2375, Oct. 2005

[5] Yingxuan Zhu, Pramod K. Varshney, Hao Chen. ICA-based fusion for color display of hyperspectral images. International Journal of Remote Sensing. vol. 32 no. 9,pp.2427-2450, 2011

[6] Ketan Kotwal , Subhasis Chaudhuri. An Optimization-Based Approach to Fusion of Hyperspectral Images. IEEE Journal of Selected Topics in Applied Earth Observations and Remote Sensing. vol. 5, no. 2, pp. 501-509, Apr 2012

[7] G. Qu, D. Zhang, and P. Yan. Information measure for performance of image fusion. Electron. Lett., vol. 38, pp. 313-315, Mar. 2002.

[8] K. Kotwal and S. Chaudhuri. A novel approach to quantitative evaluation of hyperspectral image fusion techniques. Information Fusion, vol 14,pp5-18,2013

[9] G. Qu, D. Zhang, P. Yan. Information measure for performance of image fusion. Electronics Letters 38 (2002) 313 - 315. 